ORNL/TM-2000/313

Physics Division

\title{
Workshop on Molecule Assisted Recombination and Other Processes in Fusion Divertor Plasmas September 8-9, 2000
}

\section{SUMMARY REPORT}

R. K. Janev* and D. R. Schultz

Controlled Fusion Atomic Data Center Physics Division

Oak Ridge National Laboratory

*Macedonian Academy of Sciences and Arts, Skopje, Macedonia

Date Published: November 2000

Prepared for the

Office of Fusion Energy Sciences

Budget Activity No. AT 5030100

Prepared by the

Oak Ridge National Laboratory

Oak Ridge, Tennessee 37831-6373

Managed by

UT-BATTELLE

for the

U.S. DEPARTMENT OF ENERGY

under contract DE-AC05-00OR22725 


\section{CONTENTS}

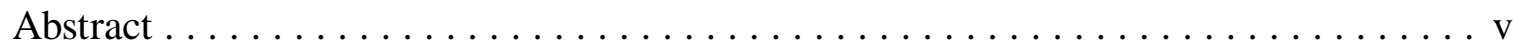

1. Introduction and Workshop Objectives $\ldots \ldots \ldots \ldots \ldots \ldots \ldots \ldots \ldots$

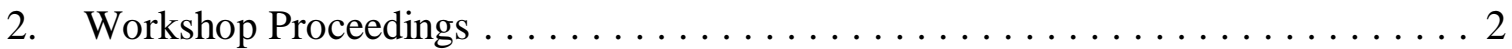

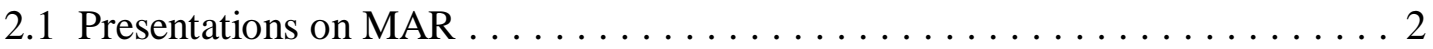

2.2 Presentations on Other Divertor Plasma Processes . . . . . . . . . . . . 8

3. Summary of Workshop Conclusions $\ldots \ldots \ldots \ldots \ldots \ldots \ldots \ldots \ldots \ldots \ldots \ldots \ldots$

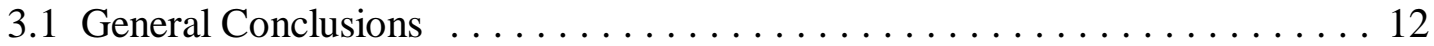

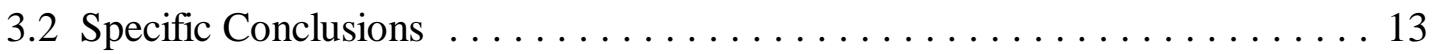

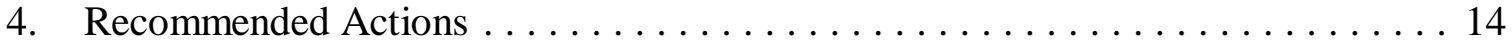

\section{Appendices}

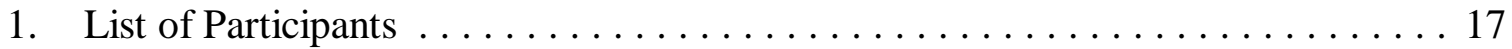

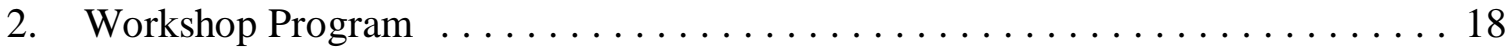




\begin{abstract}
A brief proceedings of the two-day Workshop on Molecule Assisted Recombination and Other Processes in Fusion Divertor Plasmas, organized by the ORNL Controlled Fusion Atomic Data Center on September 8-9, 2000, is presented. The conclusions and recommendations of the workshop regarding the topics discussed and the collaboration of the U.S. fusion research and atomic physics communities are also summarized.
\end{abstract}




\section{INTRODUCTION AND WORKSHOP OBJECTIVES}

Under the sponsorship of the U.S. Department of Energy Office of Fusion Energy Sciences, the ORNL Controlled Fusion Atomic Data Center (CFADC) organized on September 8-9, 2000, a workshop on "Molecule Assisted Recombination and Other Processes in Fusion Divertor Plasmas." The workshop was attended by representatives from major U.S. fusion laboratories actively involved in magnetic fusion plasma edge studies and divertor plasma modeling, as well as by atomic collision physicists involved in atomic and molecular data generation for the U.S. fusion research program. The list of workshop participants is given in Appendix 1.

The motivation for organizing the workshop was to provide a forum for stronger and direct interaction of fusion edge plasma/divertor and atomic physicists. In particular, the aim was to better understand the physics of the divertor plasma and related atomic and molecular $(\mathrm{A}+\mathrm{M})$ (collision and radiative) processes through discussion of the atomic physics content of current edge plasma and divertor modeling codes, the required $\mathrm{A}+\mathrm{M}$ data (and their availability) for enhancing the predictive power of these codes, and identification of the most urgent $\mathrm{A}+\mathrm{M}$ data related issues in divertor physics research. The organization of the workshop was in response to the presently widely recognized understanding that the achievement of the desired performance of reactor relevant divertor systems critically depends on the control of plasma conditions via atomic and molecular collision and radiative processes taking place in divertor region. Furthermore, it has been recognized that the strong interaction and collaboration between divertor plasma and atomic physicists is of paramount importance for resolving efficiently divertor optimization issues.

The concept of the two-day workshop was to devote one of the workshop days to a detailed discussion of the physics of Molecule Assisted Recombination (MAR) of divertor plasmas, with particular attention to the completeness and quality of atomic and molecular physics implemented in the MAR modeling codes. The second day was devoted to a review of the broader $\mathrm{A}+\mathrm{M}$ data needs (including particle-surface collision processes) in divertor plasma research and of the ongoing $\mathrm{A}+\mathrm{M}$ data generation efforts in the U.S. (particularly those sponsored by the DOE OFES) related to this research. This concept was reflected in the workshop program (see Appendix 2).

The specific workshop objectives regarding MAR were to:

a. determine the divertor plasma conditions in which MAR is the dominant plasma recombination mechanism;

b. identify the sensitivity of MAR modeling results on the data accuracy of the reactions involved;

c. analyze the completeness of MAR kinetics presently implemented in MAR modeling codes and assess the quality (accuracy) of employed A+M data and the needs for their improvement;

d. seek/discuss other MAR mechanisms for enhancement of volume plasma recombination (additional to those included in the present MAR scenario). 
The workshop objectives regarding the broader scope of divertor $\mathrm{A}+\mathrm{M}$ physics were to:

a. discuss and identify the $\mathrm{A}+\mathrm{M} / \mathrm{surface}$ data needs in divertor physics areas other than MAR (such as divertor plasma radiative cooling, impurity generation and transport, divertor plasma diagnostics, etc.);

b. review the status of present U.S. fusion-related $\mathrm{A}+\mathrm{M} /$ surface data generation effort (in particular its portion sponsored by the DOE OFES) in the context of its conformity with the $\mathrm{A}+\mathrm{M} /$ surface data priorities of the current U.S. fusion research program.

\title{
2. WORKSHOP PROCEEDINGS
}

The workshop was opened by Dr. D. R. Schultz, Director of the ORNL CFADC, by a welcome address and brief outline of the motivation and goals of the meeting. Dr. R. K. Janev, co-organizer of the workshop, then provided a more detailed account of the workshop objectives, putting them into the context of the needs of present-day fusion research. The work of the meeting then proceeded in accordance with the adopted Workshop Program (see Appendix 2).

\subsection{Presentations on MAR}

The presentations on MAR during the first day of the workshop are described below in the abstracts provided by the participants. We note that after the session, a general discussion took place during which various issues related to the MAR kinetics and the data availability (and their quality) were addressed. The conclusions of this discussion are summarized in Section 3.

\section{$\underline{\text { Abstracts of Presentations }}$}

\section{Molecular Activated Recombination: Mechanisms, Collision Processes and Plasma Conditions}

\author{
S. I. Krasheninnikov \\ University of California, San Diego \\ San Diego, CA
}

In experiments with detached plasmas, the plasma recombines before it reaches the targets and the recombination is the only process allowing the reduction of the plasma flux to the wall in fusion experiments without strong impurity radiation loss.

In fusion related experiments there are two main paths for plasma recombination: i) $\mathrm{e}+\mathrm{A}^{+} \rightarrow \mathrm{A}$ which includes both two- and three- body recombination of electrons and positive ions and where ions are not involved in any chemical transformations (electron-ion recombination (EIR)), and ii) pathways involving negative ions $\left(\mathrm{B}^{-}\right)$and molecular ions $\left(\mathrm{AB}^{+}\right)$and going through the channels $\mathrm{H}_{2}(\mathrm{v})+\mathrm{e} \rightarrow \mathrm{H}^{+}+\mathrm{H}$ followed by $\mathrm{A}^{+}+\mathrm{H}^{-} \rightarrow \mathrm{A}+\mathrm{H}$ and $\mathrm{A}^{+}+\mathrm{H}_{2}(\mathrm{v}) \rightarrow \mathrm{AH}^{+}+\mathrm{H}$ followed by $\mathrm{AH}^{+}+\mathrm{e} \rightarrow \mathrm{A}+\mathrm{H}$ (where $\mathrm{H}_{2}(\mathrm{v})$ is the vibrationally excited hydrogen molecule). In fusion studies the second path which cannot be initiated without impact of molecular 
hydrogen is called Molecular Activated Recombination (MAR). Notice that both paths involve rather complex dynamics of the population of electronically excited states while the second one can depend also on vibrational dynamics of the molecules involved. However, the crucial distinction between EIR and MAR is that the EIR process does not require any extra energy to start while MAR needs about $\sim 2 \mathrm{eV}$ to be pumped into vibrational levels of the molecule before it proceeds.

While the importance of the EIR processes in plasma detachment is well accepted by the fusion community, the role of MAR in fusion experiments is still under discussion and different conclusions are made based on the analysis of different experiments.

We analyze the role of EIR and MAR channels of plasma recombination in fusion related experiments by using a scaling law approach. We find that for the model relevant for a well baffled tokamak divertor geometry, the MAR effects can be important only within a rather limited zone of dimensionless heat parameter, q, proportional to the ratio of the heat flux to the plasma pressure in the upstream region in the range $\mathrm{q}<1$. For an "optically transparent" gas box model, relevant for divertor simulators and slot divertors, we find that the MAR effects are important at $\mathrm{q} \sim 1$. Our findings may explain the differences in the experimental results from the weakly baffled C-Mod inner leg, the divertor simulator NAGDIS-II, and the well baffled ASDEX-U divertor.

\section{Collisional Radiative Kinetics of Molecular Assisted Recombination in Edge Plasma \\ A Yu. Pigarov \\ University of California, San Diego \\ San Diego, CA}

Recombination of hydrogen plasma in the divertor volume is a simple explanation for divertor plasma detachment phenomena experimentally observed on many tokamaks. ${ }^{1}$ The presence of vibrationally excited molecules in detached plasma provides an additional mechanism for plasma recombination, the so-called, molecular assisted recombination (MAR). ${ }^{2}$

The kinetics of cascade excitation of vibrational states of hydrogen molecule and molecular ion, dissociation, and numerous reactions has been studied with the collisional radiative atomic molecular data (CRAMD) code. ${ }^{2}$ This code solves a system of coupled rate balance equations for: (i) vibrational levels of ground state molecule $\mathrm{H}_{2}\left(\mathrm{x}^{1} \Sigma_{\mathrm{g}}^{+}\right)$; (ii) the few lowest electronically and vibrationally excited states of $\mathrm{H}_{2}$; (iii) electronically excited states and all vibrational levels of the ground state of molecular ion $\mathrm{H}_{2}^{+}$; (iv) the negative ion $\mathrm{H}^{-}$; and (v) electronically excited states of hydrogen atom. A complete set of atomic physics data has been developed. The quasi-stationary, quasi-homogenous plasma approximation is used.

The calculations show that the effective rate coefficient for MAR is about $10^{-10} \mathrm{~cm}^{3} / \mathrm{s}$ and it exceeds the rate coefficient for usual electron-ion recombination (EIR) in a wide range of plasma parameters. The dependence of the MAR rate coefficient on plasma parameters is 
discussed. A comparison of the MAR rate coefficient with rate coefficients for molecular dissociation and ionization and EIR is given.

The sensitivity of the MAR rate on the accuracy and completeness of atomic physics data is discussed. A three-times difference in predicted MAR rate coefficient was obtained under different model assumptions on state-to-state cross sections for atomic-to-molecular ion conversion. Completeness of the MAR model requires incorporation of detailed data on the molecular ion, since the cross sections for ionization and dissociative recombination of $\mathrm{H}_{2}^{+}$ as well as the quantum state of dissociation products depend strongly on vibrational excitation of the ion.

High concentrations of neutral atoms in detached divertor plasma cause strong re-absorption of Lyman radiation. The effect of plasma radiation opacity on the MAR rate coefficient is considered in terms of radiation escape factors.

The results of the CRAMD modeling of Lyman and Balmer line intensities measured from detached divertor plasma in ALCATOR C-Mod tokamak are presented. For a two-emissionvolumes model of the divertor plasma, the CRAMD code composes the line spectra from different mechanisms for excited states population (MAR, EIR, and excitation of ground state atoms). The analysis shows that MAR should be involved in explaining the measured spectra and that the contribution of MAR to the plasma particle sink in the divertor is significant.

${ }^{1}$ S. I. Krasheninnikov, A. Yu. Pigarov et al, Phys. Plasmas 4, 1638 (1997).

${ }^{2}$ A. Yu. Pigarov, S. I. Krasheninnikov, Phys. Lett. A 222, 251 (1996).

\section{A\&M Database for MAR and Divertor Plasma Modeling in Neutral Particle Codes: Data Status and Needs Daren Stotler \\ Princeton Plasma Physics Laboratory \\ Princeton, NJ}

The collisional radiative model used to treat the $\mathrm{H}_{2}$ in the EIRENE Monte Carlo neutral transport code is described by Greenland. ${ }^{1}$ The data used in this model are enumerated by Greenland and Reiter. ${ }^{2}$ The paper by Fantz et al. ${ }^{3}$ gives a recent application of this model in support of the measurement of the vibrational distribution of $\mathrm{H}_{2}$ in the ASDEX-Upgrade tokamak experiment.

A key conclusion of Greenland ${ }^{1}$ is that establishing the validity of a given collisional radiative model involves more than just examining the equilibrium timescales of the component processes. The application of this analysis to the case of electron impact on hydrogen reveals that a valid collisional radiative model must treat all vibrationally excited states, 14 in the paper, as "slowly evolving" species (i.e., their transport must be explicitly computed). Doing this has a significant impact on the results of neutral particle transport simulations, as is demonstrated by the results shown by Fantz et al. ${ }^{3}$ 
Investigation of the MAR reaction chain in experimentally relevant simulations with the EIRENE and B2-EIRENE codes $^{3}$ shows that the recombination contribution made by MAR is a few percent in detached divertor conditions and less than $1 \%$ in attached operation. The results were shown to be insensitive to the vibrational distribution of molecules coming from the walls.

${ }^{1}$ P. T. Greenland (to appear in J. Nucl. Mater., 2000).

${ }^{2}$ P. T. Greenland and D. Reiter, "The role of molecular hydrogen in plasma recombination," Jul-3258 ISSN 0944-2952 (Forschungszentrum Julich) (1996).

${ }^{3}$ U. Fantz et al. (to appear in J. Nucl. Mater., 2000).

\section{Dissociative Electron Attachment on Hydrogen Isotope Molecules: Status}

Jo Wadehra

Wayne State University

Detroit, MI

At the Workshop on Molecule Assisted/Activated Recombination and Other Processes in Divertor Plasmas, I presented theoretical cross sections for dissociative electron attachment to $\mathrm{H}_{2}$ and its five heavier isotopic variants (HD, $\left.\mathrm{HT}, \mathrm{D}_{2}, \mathrm{DT}, \mathrm{T}_{2}\right)$. The dependence of these cross sections on the initial vibrational excitation of the molecule was also investigated and presented. Various cross sections and the corresponding rates were fitted to simple analytical functions. These functions can be included in the computer codes to assess the effect of the electron attachment processes in the divertor modeling. At the Workshop I also presented the results of our calculations of cross sections for vibrational excitation and for pure dissociation of $\mathrm{H}_{2}$.

\section{Resonance Processes in e- $\mathrm{H}_{2} / \mathrm{HD} / \mathrm{D}_{2}$ Collisions: Dissociative Attachment and Dissociation from Vibrationally Excited States \\ Ilya Fabrikant \\ University of Nebraska \\ Lincoln, NB}

Quasiclassical version of the non-local complex potential theory allows us to describe the dissociative electron attachment to the rovibrationally ground and excited states of the $\mathrm{H}_{2}$ molecule and its isotopic substitutes. The theory also allows us to calculate vibrational transitions with the extension to the vibrational continuum (resonant dissociation). We find that the rotational enhancement of the dissociative attachment process is much weaker than the vibrational enhancement. The isotope effect is much stronger for lower vibrational states. Both findings can be interpreted in terms of Franck-Condon factors and survival probabilities of the intermediate resonant state of $\mathrm{H}_{2}^{-}$. 


\section{Collisional-Radiative Model For Molecular Hydrogen}

Takashi Fujimoto

Kyoto University, Kyoto, Japan

Keiji Sawada

Shinshu University

In the course of our spectroscopy of Balmer lines on our WT-3 tokamak plasma, we found that the observed intensities of these lines were strongly influenced by the presence of molecular hydrogen. We estimated the contribution from dissociative excitation of molecular hydrogen to the atomic line intensities. We thus succeeded in determining densities of atoms, molecules and electrons from the three observed line intensities, i.e., Balmer alpha, beta and gamma lines, as functions of the radial position. We also estimated the transport fluxes of atoms and molecules. In our analysis, we effectively assumed that all the molecular hydrogen was in its electronic, vibrational and rotational ground state. Obviously this assumption is not correct, but our above analysis gave rather reasonable results.

For divertor plasmas, recombination processes should be included. Especially recombination starting from molecular ions is a key to understanding of the overall picture of the plasma. MAR is a kind of a "new face" of recombination mechanisms, and its effect should be quantitatively evaluated. For that purpose, we should have a good knowledge about (1) the vibrational population distribution of neutral hydrogen when it is released from the wall, (2) vibrational excitation cross section by electrons, (3) charge exchange cross sections between neutral molecules and protons producing molecular ions, where vibrational states should be resolved both for neutrals and ions, and (4) various cross sections concerning molecular ions in all the vibrationally ground and excited levels. Among the above, (2) was addressed by Dr. Wadehra at the meeting, (3) was also done by Dr. Krstic. Dr. Takagi has comprehensive calculated data for (4). Until now, rotational excitation and its effect on the dynamics have never been addressed except for the Takagi's calculation. Since rotational excitation of neutral and ionized molecules by atoms (molecules) and ions should be effective, this problem should be considered seriously.

\section{Charge Transfer, Vibrational Excitation And Dissociation In Slow Hydrogen Ion-Molecule Collisions}

P.S. Krstic

Oak Ridge National Laboratory

Oak Ridge, TN

We presented extensive calculations of charge exchange, vibrational excitation and dissociation in collisions of $\mathrm{H}^{+}$with $\mathrm{H}_{2}(\mathrm{v})$, obtained within the quantum-mechanical IOSA model. The $a b$ initio potential energy surfaces for the $\mathrm{H}_{3}{ }^{+}$ion were employed and the corresponding coupled equations were solved for all 34 vibrational levels (corresponding to the asymptotic arrangements $\mathrm{H}^{+}+\mathrm{H}_{2}(\mathrm{v})$ and $\left.\mathrm{H}+\mathrm{H}_{2}^{+}(\mathrm{v})\right)$ and for 66 discrete continuum states. We also described some of the underlying dynamical mechanisms for the above processes and presented the relevant cross section results from the threshold to about $10 \mathrm{eV}$. The particle 
exchange (heavy particle rearrangement) channel, however, was not included in these calculations which limits their validity to the region above $1.5-2 \mathrm{eV}$. Also, the full convergence of the calculations seems to require a larger number of discrete continuum states. Comparison of vibrational excitation cross sections agrees well with the existing experimental data for low v-values, while the charge exchange cross sections in the energy range above 2 $\mathrm{eV}$ are in qualitative agreement with the recent classical-trajectory-surface-hopping (TSH) of Ichihara. Inclusion of the particle exchange effects as well as improvement of the convergence of the calculation by increasing the basis set is underway.

\section{Hydrogen Atom, Ion, and Molecule Isotopomer Collisions: Rearrangement, Electron Transfer, and Excitation Phillip C. Stancil \\ University of Georgia, Athens Athens, GA}

A survey of existing data for collisions of isotopes of hydrogen atoms, ions, and molecules was conducted. The survey was limited to atom (ion) - diatom collisions and to energies less than about $100 \mathrm{eV}$. The processes included particle-rearrangement, electron transfer, and rovibrational excitation, with a particular emphasis on state-to-state (or state-selected) data. Since the last survey (Linder, Janev, and Botero, 1995), a small number of investigations for deuterium ion-diatom systems have been performed, with only one involving state-resolved data. Data for the dissociative channel and for neutral atom-molecular ion collisions are rare, while no data involving tritium ion-diatom systems was found. For neutral atom-diatom collisions, a number of recent state-to-state studies on both particle-rearrangement and rotational excitation have been reported for deuterium, and in the former case, tritium collision systems. In summary, while some progress has been made since the last survey, the database involving hydrogen isotope collisional processes, both total and state-resolved, is far from complete.

\section{Alternative Mechanisms in MAR \\ R. K. Janev* \\ Oak Ridge National Laboratory, Oak Ridge, TN (visiting); \\ *Macedonian Academy of Sciences and Arts, Skopje, Macedonia}

The enhancement of MAR in divertors via mechanisms different than the standard ionconversion and negative-ion formation channels would be highly beneficial from the point of view of divertor operation. Two such alternative mechanisms were analyzed. One is based on the hypothetical existence (formation) of Rydberg $\mathrm{H}_{2}$ molecules in the cold divertor regions, the dissociative electron attachment of which should have huge $\left(\sim 10^{-7} \mathrm{~cm}^{3} / \mathrm{s}\right)$ rate coefficients. $\mathrm{H}_{2}$ molecules in Rydberg states have been observed in some plasmas with parameters close to those in fusion divertors. Dissociative electron attachment on a small fraction of Rydberg $\mathrm{H}_{2}$ in the divertor would strongly enhance the negative ion MAR channel.

The other alternative MAR mechanism is based on the presence of hydrocarbon $\left(\mathrm{C}_{x} \mathrm{H}_{\mathrm{y}}\right)$ 
impurities in divertor plasma (which is a reality for almost all presently operating divertors). The mechanism consists in proton charge exchange with a $\mathrm{C}_{\mathrm{x}} \mathrm{H}_{\mathrm{y}}$ molecule, followed by the dissociative recombination of produced $\mathrm{C}_{\mathrm{x}} \mathrm{H}_{\mathrm{y}}{ }^{+}$ions with plasma electrons. Since the products of $\mathrm{e}+\mathrm{C}_{\mathrm{x}} \mathrm{H}_{\mathrm{y}}^{+}$recombination are again hydrocarbon molecules, the charge exchangedissociative recombination cycle can be (multiplicatively) repeated with the hydrocarbon fragments of the previous cycle. The mechanism, thus, exhibits a catalytic property which makes its effectiveness very high. Calculations show that $\sim 5-6 \%$ of $\mathrm{C}_{\mathrm{x}} \mathrm{H}_{\mathrm{y}}$ in the divertor will have the same plasma recombining effect as the entire molecular hydrogen gas.

The potential role of $\mathrm{H}_{3}{ }^{+}$ions in the divertor plasma volume recombination was also discussed.

\subsection{Presentations on Other Divertor Plasma Processes}

The second day of the workshop was devoted to atomic processes in divertor plasmas related to other (non-MAR) aspects of these plasmas such as radiative cooling, ionization balance, divertor plasma diagnostics and interactions of plasma particles with material surfaces. The session ended with a general discussion on various atomic and particle-surface interaction issues of the current divertor plasma research. The main conclusions of this discussion are summarized in Section 3.

\section{$\underline{\text { Abstracts of presentations }}$}

\section{Overview of Important A\&M Divertor Plasma Processes \\ R. K. Janev* \\ Oak Ridge National Laboratory, Oak Ridge, TN (visiting); \\ *Macedonian Academy of Sciences and Arts, Skopje, Macedonia}

The atomic and molecular collision processes in divertor plasmas were reviewed from the point of view of their role in divertor plasma cooling, power and particle exhaust from divertor region, divertor plasma diagnostics, and neutral particle and impurity transport in divertors. In particular, the radiative plasma losses due to hydrogen and impurities were analyzed (with identification of the main participating atomic/molecular processes), the processes involved in plasma momentum dissipation in the divertor were highlighted, the processes involved in the emission and charge exchange beam diagnostics of divertor plasmas were enumerated, etc. A brief account of the status of the corresponding required databases for these plasma studies was also given, emphasizing the data gaps and deficiencies. The particle-surface interaction processes in the divertor region were also reviewed and the status of our understanding of their physical mechanisms and methods of their description was briefly presented. 


\section{Ionization and Recombination in Electron-Ion Scattering}

M. S. Pindzola, J. P. Colgan, and F. J. Robicheaux

Auburn University

Auburn, AL

D. C. Griffin and D. M. Mitnik

Rollins College

Winter Park, FL

N. R. Badnell, C. P. Ballance, and H. P. Summers

University of Strathclyde

Glasgow, UK

An electron-ion scattering database for ionization and recombination is now available at the Oak Ridge National Laboratory's Controlled Fusion Atomic Data Center (see wwwcfadc.phy.ornl.gov). The general format contains final state resolved Maxwellian rate coefficients as a function of electron temperature, as developed for experimental spectroscopic diagnostics at the Joint European Torus. Direct ionization rate coefficients are available for all $\mathrm{Be}, \mathrm{B}, \mathrm{C}, \mathrm{N}, \mathrm{O}$, and $\mathrm{Ne}$ ions, while direct and indirect ionization rate coefficients are available for $\mathrm{Na}$-like and $\mathrm{Mg}$-like $\mathrm{Ti}, \mathrm{Cr}, \mathrm{Fe}$, and $\mathrm{Ni}$ ions. Dielectronic recombination rate coefficients are available for all $\mathrm{He}, \mathrm{Li}, \mathrm{Be}, \mathrm{B}, \mathrm{C}, \mathrm{N}, \mathrm{O}$, and $\mathrm{Ne}$ ions, while dielectronic recombination rate coefficients are available for $\mathrm{H}$-like through $\mathrm{Ne}$-like $\mathrm{Ar}$ and $\mathrm{Fe}$ ions.

Recent time-dependent close-coupling and R-matrix pseudo-state calculations for the total electron-impact ionization cross sections of $\mathrm{He}$ and $\mathrm{Li}^{+}$are found to be in good agreement with experimental measurements. State selective ionization and recombination theoretical predictions remain unconfirmed by atomic experiments.

\section{Electron-Impact Excitation of Impurity Ions \\ Donald C. Griffin \\ Rollins College \\ Winter Park, FL}

In this presentation, we summarized the electron-impact excitation data that have been stored at the CFADC Internet site at ORNL as part of a collaborative effort between Rollins College, Strathclyde University, and Auburn University. They were generated from large-scale Rmatrix close-coupling calculations and consist of energy levels, radiative rates, and effective collision strengths in the ADAS format. The applications of these data to collisional-radiative modeling of laboratory and astrophysical plasmas were briefly discussed. As an example, we also presented the results our R-matrix with pseudo state calculations of the electron-impact of $\mathrm{C}^{3+}$ and $\mathrm{O}^{5+}$, where the effects of coupling to the target continuum states has a significant effect on the cross sections for transitions to the 2s4l excited levels. 


\section{Overview of Electron-Ion Collision Experiments at ORNL \\ M. E. Bannister \\ Oak Ridge National Laboratory \\ Oak Ridge, TN}

An introduction to the electron-ion collision experiments performed at the ORNL Multicharged Ion Research Facility (MIRF) was presented. The merged electron-ion beams energy-loss (MEIBEL) experiment, a collaboration with the research group of Gordon Dunn of JILA, measures absolute near-threshold excitation cross sections for multicharged ions of interest to fusion. Recent results on $\mathrm{C}^{3+}$ and $\mathrm{He}^{+}$demonstrate the ability of the apparatus to accurately measure allowed transitions, while data on spin-forbidden excitations in $\mathrm{Si}^{2+}$ and $\mathrm{Ar}^{6+}$ show the capability of investigating dielectronic resonance structures as well. The electron-ion crossed beams experiment was also highlighted, including a summary of the range of ions for which single ionization cross sections have been measured with the apparatus. The crossed-beams experiment is also used to study electron-impact dissociative excitation (DE) and dissociative ionization (DI) of molecular ions. Further details on the experiments, as well as all the published data, can be found at: http://wwwcfadc.phy.ornl.gov/meibel/ and http://www-cfadc.phy.ornl.gov/xbeam/.

\section{Electron Impact Ionization of Divertor Plasma Molecular Impurities Y.-K. Kim \\ National Institute of Standards and Technology \\ Gaithersburg, MD}

Electron-impact ionization cross sections for molecules and their ions can be estimated using the binary encounter dipole (BED) model and its simplified versions. Cross sections based on these ab initio models are expressed by a simple analytic formula for each molecular orbital. The outline of the theory, necessary molecular constants to calculate ionization cross sections, and comparisons to available experimental data can be found at a public Website: http://physics.nist.gov/ionxsec. The Website contains cross sections for almost 70 molecules, including most hydrocarbons relevant to magnetic fusion plasma modeling. Please contact kim@nist.gov for more information.

\section{CTMC Charge Exchange Cross Sections: n-Scaling Ronald E. Olson University of Missouri, Rolla Rolla, MO}

The classical trajectory Monte Carlo method was used to calculate total and final $\left(n_{f}\right)$ state selective electron capture cross sections for stripped ions ranging from $\mathrm{He}^{2+}$ to $\mathrm{Ne}^{10+}$ incident on $\mathrm{H}\left(n_{i}=1-4\right)$ and $\mathrm{Li}(2 \mathrm{~s}$ and $2 \mathrm{p})$ targets at collision energies of $1-100 \mathrm{keV} / \mathrm{u}$. Scaling laws for state-selective electron capture cross sections as a function of collision energy, initial and final bound electron states, and projectile charge were given. The scaling rules reduce the state-selective cross sections to distinct universal curves. The curves allow one to predict 
electron capture cross sections in low-energy regions where experimental data are limited or non-existent. Reference: K. R. Cornelius, K. Wojtkowski, and R. E. Olson, J. Phys. B 33, 2017 (2000).

\author{
Overview of Ion-Atom/Molecule Collision Experiments at ORNL \\ C. C. Havener \\ Oak Ridge National Laboratory \\ Oak Ridge, TN
}

At the ORNL Multicharged Ion Research Facility (MIRF) (visit www-cfadc.phy.ornl.gov) ion-atom and ion-molecule experiments provide data that is relevant to fusion plasmas and that test our current understanding of low energy collisions. Scaling laws which are often used to estimate electron capture cross sections at $\mathrm{keV} / \mathrm{amu}$ collision energies are not appropriate at eV/amu energies. At MIRF total single and double electron capture cross sections for a variety of multicharged ions on atomic and molecular gases are measured using a gas cell. Such measurements for $\mathrm{B}^{3+}+\mathrm{H}_{2}$ in the keV/amu energy range provide a benchmark to recent coupled channel molecular-orbital calculations. To address low energy collisions, the ionatom merged-beams apparatus provides benchmark absolute total electron capture cross sections for collisions between multicharged ions and ground state $\mathrm{H}$ or $\mathrm{D}$ in the energy range from $.02 \mathrm{eV} / \mathrm{amu}$ to $5000 \mathrm{eV} / \mathrm{amu}$. For example, our measurements for $\mathrm{C}^{+}$ions with $\mathrm{H}$ have shown dramatic differences in the total cross section for collisions with metastable versus ground state ions. For $\mathrm{C}^{3+, 4+}$ our measurements provide a new benchmark for normalization of state-selective data and show structure which is not reproduced by current theory. Measurements with $\mathrm{Si}^{4+}+\mathrm{D}$ provide evidence of a strong isotope effect at $\mathrm{eV} / \mathrm{amu}$ energies. The merged-beams technique will soon be expanded to include multicharged ion collisions with a variety of neutrals, e.g., Li, Na, and Fe. At eV/amu energies and below, such collisions systems are virtually unexplored.

\title{
Ion-Surface Interaction Studies at the ORNL Multicharged Ion Research Facility V. A. Morozov \\ Oak Ridge National Laboratory \\ Oak Ridge, TN
}

An overview was provided of present and planned experimental activities in the area of ionsurface scattering at ORNL-MIRF. Using a recently developed floating scattering chamber, deceleration of ion beams prior to surface impact down to a few $\mathrm{eV}$ has been achieved. A TOF system permits energy and charge state analysis of projectiles and secondary particles $120^{\circ}$ backscattered from solid targets. A movable electrostatic spherical sector analyzer permits in addition measurements of energy spectra of electrons emitted during projectile interactions with the surface. The apparatus has already been used to study neutralization of multicharged projectiles during binary collisions with isolated atoms in metal and insulator surfaces. We have recently initiated studies of chemical and physical sputtering processes relevant to divertor and edge plasmas of fusion devices. While the initial focus will be on sputtering of ATJ graphite by low energy $\mathrm{H}^{+}, \mathrm{D}^{+}, \mathrm{He}^{+}$, multicharged impurity ions, and 
molecular species (e.g., light hydrocarbons), future studies with liquid and solid $\mathrm{Li}$ overcoatings will be possible as well, in an attempt to elucidate the beneficial effects of $\mathrm{Li}$ pellet injection and laser ablation demonstrated in the final days of TFTR, and to support liquid Li limiter and divertor experiments on present devices such as CDX-U.

\section{SUMMARY OF WORKSHOP CONCLUSIONS}

The extensive discussion sessions held after the presentation sessions of the first and second workshop days have addressed many general and specific questions related to the role of atomic and molecular processes in fusion divertor plasmas, the proper inclusion of these processes in the divertor plasma studies (e.g., plasma modeling and diagnostics), the quality of atomic data information presently used in plasma modeling codes and diagnostic methods, the data availability and data needs for various divertor plasma studies, the status of data information exchange between data producers and users, etc. The main conclusions of these discussions can be summarized as follows.

\subsection{General Conclusions}

(1) The Workshop participants adhere to and share the widespread and well founded recognition by the fusion community that one of the critical issues of current fusion reactor development is the successful design and operation of the reactor divertor system (for resolving the critical problems of thermal power and particle exhaust, reduction of power and particle flux loads on the divertor plate materials, efficient impurity control, etc.). Furthermore, it was recognized that in the most recent divertor concepts (as implemented, e.g., in the ITER EDA and in the presently most advanced fusion devices) the trust in the achievement of the desired divertor functions and performance is placed on the atomic and molecular (collision and radiative) processes and on their dominant role in determining the physical conditions of divertor plasmas. In particular, the atomic and molecular processes in divertor plasmas may critically influence the plasma energy balance (through various plasma cooling mechanisms), plasma momentum distribution (and dissipation), plasma ionizationrecombination equilibrium regime, the conditions for plasma-wall interaction processes (and, thereby, the impurity generation, hydrogen recycling, etc.).

(2) Under the conditions when atomic and molecular physics plays such an important, and in many respects decisive, role in the divertor plasma physics research, the strong interaction and even direct collaboration of atomic and divertor plasma physicists becomes an imperative. Such cooperation enables an efficient and rational approach in resolving the outstanding problems of optimization of the design and operational performance of a fusion reactor divertor, also for a successful carrying out and interpretation of the experimental and computational (modeling) divertor studies on the presently operating magnetic fusion devices.

(3) Joint collaborative efforts between atomic and divertor plasma researchers would be particularly useful in the areas of divertor plasma and neutral particle modeling, divertor plasma diagnostics, divertor plasma cooling studies and plasma-wall interaction effects. The

present level of interaction between the atomic physics and fusion research communities in 
the above areas is, in general, indirect (e.g., via DOE OFES, or ORNL CFADC) and the time correlation between the data generation efforts and the data needs is not always adequate.

(4) The present Workshop has demonstrated to the full extent the usefulness of working-level meetings between atomic and plasma researchers on well focused topics (in the present case the MAR physics), and also the usefulness of a detailed information exchange on the $\mathrm{A}+\mathrm{M}$ data needs and availability in the broader scope of fusion plasma research (in the present case the divertor physics). Continuation of the practice of such workshops, each time focused on a different topical area, at which atomic and plasma physicists discuss in detail a specific set of research problems and agree on certain courses of action, is highly desirable and the workshop participants recommend it strongly.

(5) The ORNL Controlled Fusion Atomic Data Center has been (since its inception) and remains the focal point of the U.S. effort on collection, critical assessment and generation (to a significant extent) of atomic and molecular data for the U.S. fusion program (and beyond). In the present situation, when the strengthening of the interaction between atomic and fusion research, particularly in the area of divertor plasma physics, is becoming essential for ensuring an efficient and rational approach to solving plasma research issues containing a strong atomic physics component, the role of the ORNL CFADC in catalyzing this interaction and providing even the necessary level of effort, and coordination could be very important. A series of CFADC organized workshops, such as the present one, as well as the numerical $\mathrm{A}+\mathrm{M}$ database maintained by CFADC, provide basic tools by which CFADC could exercise such a role.

\subsection{Specific Conclusions}

\section{Regarding MAR processes}

(1) The kinetic reaction schemes presently employed in the collisional-radiative codes for modeling MAR, generally adequately describe the relevant atomic physics for the case of a pure hydrogen plasma. There are, however, a number of key reactions (such as proton charge exchange on vibrationally excited $\mathrm{H}_{2}$ ) for which the required database is still not available with sufficient accuracy. The quality (accuracy) of the A+M collisional data implemented in the modeling codes differs from code to code and comparison of their results for the same plasma conditions is at present not possible.

(2) The most important gaps in the A+M database for MAR modeling are in the area of vibrational and rotational kinetics. The data for the process of vibrational excitation of $\mathrm{H}_{2}(\mathrm{v})$ via the resonant $\mathrm{H}_{2}{ }^{+}$state are still not implemented in all MAR modeling codes. The recent observations of high $\mathrm{H}_{2}^{+}$rotational temperatures in some divertor plasmas indicate that ro-vibrational kinetics has to be implemented in the MAR modeling codes (and not only the vibrational one). This raises the question of a major upgrading of the existing A+M databases with collisional data for ro-vibrationally excited $\mathrm{H}_{2}$ (and $\mathrm{H}_{2}^{+}$), which, for most of the processes, presently do not exist. Similar upgrading is required also for the modeling codes. 
(3) Reactor relevant studies of MAR should consider the collision processes of heavier hydrogen isotopes $\mathrm{D}_{2}$, DT, and $\mathrm{T}_{2}$. There are several reactions in the MAR kinetic scheme which proceed via intermediary states (e.g., the dissociative electron attachment, e-impact vibrational excitation) and which may exhibit an isotope effect. The collisional data available for $\mathrm{H}_{2}(\mathrm{v})$ cannot be in such cases directly applied to the same processes with heavier isotopes. The isotopic shift of vibrational levels in different hydrogen molecule isotopes requires also carrying out new calculations for all the processes in which the internal energy of the reactants plays a critical role (e.g., in the ion-conversion reaction). Particle exchange reactions in isotopic mixtures are expected to play an important role (in the temperature region about 1 $\mathrm{eV}$ ) and there is at present no cross section data information for such reactions involving D and $\mathrm{T}$ isotopes.

\section{Regarding other (non-MAR) processes}

(1) Elastic, charge exchange, and momentum transfer processes between plasma ions and divertor neutrals are the main plasma momentum dissipation processes in the divertor. The database for these processes for all hydrogen ion-atom (molecule) isotopomers has recently been established by the ORNL CFADC and has a very high accuracy. For the neutral particle transport studies in the divertor, this database constitutes one of the basic inputs.

(2) The theoretical and experimental data generated in the last few years by the U.S. DOE OFES contractors for the electron-impact inelastic processes involving plasma impurity atoms, ions and molecules, as well as for the heavy particle collisions at low energies, adequately address the present needs of the fusion research, including that of fusion divertors. The accuracy of these data is sufficient for the needs of fusion applications. Use of cross section scaling properties (with respect to atomic or interaction parameters) would make easier the implementation of the results of massive cross section computations into the plasma application codes, but this approach is not always possible.

(3) Particle-surface collision processes are important source/sink terms in the impurity and neutral particle transport equations, but also participate in the vibrational kinetics of the divertor molecular gas. Knowledge of the vibrational distribution of hydrogen molecules created on (or released from) the divertor wall/plate surfaces is of paramount importance for the overall divertor plasma chemistry (particularly that involved in MAR).

\section{RECOMMENDED ACTIONS}

The Workshop participants recommend the following specific actions be undertaken for enhancing the interaction between fusion and atomic physics communities in resolving more effectively some of the critical fusion research.

(1) In view of the demonstrated extreme usefulness of the present Workshop, it is hereby strongly recommended that this type of workshop, with a well focused topical part and a more broader $\mathrm{A}+\mathrm{M}$ data reviewing part, be held annually with their organization entrusted to the ORNL CFADC. A Summary Report of these workshops should be published by the 
ORNL CFADC and distributed to the relevant institutions interested in the workshop outcomes and conclusions.

(2) The presentations of the topical part of the Workshop bear scientific importance and it is recommended that they be published in a scientific journal. The ORNL CFADC is asked to take over the editorial responsibilities in the preparation of such a publication and to select the publisher (journal). The IAEA APID series and Physica Scripta (Topical Issues series) are recommended as a possible publication medium for the workshop presentations.

(3) It is recommended that the MAR modelers and atomic data generation groups undertake immediate actions to identify the most urgent $\mathrm{A}+\mathrm{M}$ data needs for the MAR kinetics and define joint collaborative projects for improving the atomic physics of MAR modeling codes. Inclusion of rotational kinetics in MAR is one of the most important areas in which such joint effort is urgently required. 


\begin{abstract}
APPENDIX 1
Workshop on Molecule Assisted Recombination and Other Processes in Fusion Divertor Plasmas Oak Ridge National Laboratory
\end{abstract}

Sept. 8-9, 2000

\title{
$\underline{\text { Participants }}$
}

Mark E. Bannister, Oak Ridge National Laboratory, Oak Ridge, TN James P. Colgan, Auburn University, Auburn, AL Michael D. Crisp, Office of Fusion Energy Sciences, DOE, Germantown, MD Gordon H. Dunn, Joint Institute for Laboratory Astrophysics, Boulder, CO Ilya Fabrikant, University of Nebraska, Lincoln, NB Takashi Fujimoto, Kyoto University, Kyoto, Japan Donald C. Griffin, Rollins College, Winter Park, FL Charles C. Havener, Oak Ridge National Laboratory, Oak Ridge, TN Ratko Janev, Macedonian Academy of Sciences and Arts, Skopje, Macedonia Yong-Ki Kim, National Institute of Standards and Technology, Gaithersburg, MD Sergei Krasheninnikov, University of California, San Diego, CA Herb F. Krause, Oak Ridge National Laboratory, Oak Ridge, TN Predrag Krstic, Oak Ridge National Laboratory, Oak Ridge, TN Fred W. Meyer, Oak Ridge National Laboratory, Oak Ridge, TN Victor A. Morozov, Oak Ridge National Laboratory, Oak Ridge, TN Jens Mrogenda, University of Muenster, Muenster, Germany Ronald E. Olson, University of Missouri, Rolla, MO Sergei Ovchinnikov, Oak Ridge National Laboratory, Oak Ridge, TN Alexander Pigarov, University of California, San Diego, CA Michael S. Pindzola, Auburn University, Auburn, AL Milun Rakovic, Oak Ridge National Laboratory, Oak Ridge, TN Carlos Reinhold-Larsson, Oak Ridge National Laboratory, Oak Ridge, TN

David R. Schultz, Oak Ridge National Laboratory, Oak Ridge, TN

Phillip C. Stancil, University of Georgia, Athens, GA

Daren P. Stotler, Princeton Plasma Physics Laboratory, Princeton, NJ Jo Wadehra, Wayne State University, Detroit, MI

Jianguo Wang, University of Georgia, Athens, GA 


\section{APPENDIX 2 \\ Workshop on Molecule Assisted Recombination and Other Processes in Fusion Divertor Plasmas \\ September 8-9, 2000 \\ Oak Ridge National Laboratory}

Friday, September 8, 2000 - Molecule Assisted Recombination

8:30-9:00 Coffee

9:00-9:15 Schultz, Janev - Welcome, Workshop objectives

9:15-9:45 Krasheninnikov - MAR: Mechanisms, collision processes and plasma conditions

9:45-10:15 Pigarov - Collisional radiative kinetics of MAR in edge plasma

10:15-10:45 Coffee

10:45-11:15 Stotler - A\&M database for MAR and divertor plasma modeling in neutral particle codes: data status and needs

11:15-11:45 Wadehra - Dissociative electron attachment on hydrogen isotope molecules: status

11:45-12:15 Fabrikant - Resonance processes in $\mathrm{e}-\mathrm{H}_{2} / \mathrm{HD} / \mathrm{D}_{2}$ collisions: dissociative attachment and dissociation from vibrationally excited states

12:15-2:15 Lunch

2:15-2:45 Fujimoto - Collisional-radiative model for molecular hydrogen

2:45-3:15 Krstic - Charge transfer, vibrational excitation and dissociation in slow hydrogen ion-molecule collisions

3:15-3:30 Stancil - Particle interchange in slow (hydrogenic) ion-molecule collisions

3:30-4:00 Coffee

4:00-4:30 Janev - Alternative mechanisms in MAR

4:30-5:30 Discussion - Krasheninnikov, Janev

6:30 Dinner reception 
Saturday, September 9, 2000 - Other Processes in Divertors

8:30-9:00 Coffee

9:00-9:30 Janev - Overview of important A\&M divertor plasma processes

9:30-9:45 Pindzola - Electron-impact excitation, ionization of low-q plasma impurity ions

9:45-10:00 Griffin - Recombination of low-q plasma impurity ions with electrons

10:00-10:30 Coffee

10:30-10:50 Bannister - Overview of electron impact collision experiments at ORNL

10:50-11:10 Kim - Electron impact ionization of divertor plasma molecular impurities

11:10-12:15 Discussion, Dunn

12:15-2:15 Lunch

2:15-2:35 Olson - CTMC charge exchange cross sections: $n$-scaling

2:35-2:55 Havener - Overview of ion-atom/molecule collision experiments at ORNL

2:55-3:15 Morozov - Overview of particle-surface collision processes at ORNL

3:15-3:45 Coffee

3:45-4:45 Discussion, Krstic

4:45-5:00 Recommendations, summary, Schultz 
ORNL/TM-2000/313

\section{Internal Distribution}

1. M. E. Bannister

2. S. Datz

3. C. C. Havener

4. J. T. Hogan

5. R. C. Isler

6. H. F. Krause

7. P. S. Krstic

8. J. H. Macek

9. F. W. Meyer

10. S. L. Milora

11. P. Mioduszewski

12. V. A. Morozov
13. J. Mrogenda

14. S. Yu. Ovchinnikov

15. F. M. Ownby

16. M. Rakovic

17. C. O. Reinhold

18. D. R. Schultz

19. C. R. Vane

20. Fusion Energy Division Library

21. ORNL Central Research Library

22. Laboratory Records

\section{External Distribution}

23. Office of Scientific and Technical Information, P.O. Box 62, Oak Ridge, TN 37831

24. K. Behringer, Lehrstuhl für Experimentelle Plasmaphysik, Memmingerstrasse 6, 86135 Augsburg Germany

25. L. M. Blush, University of California, San Diego, FERP, 9500 Gilman Dr., Mail Code 417, La Jolla, CA 92093-0417

26. J. N. Brooks, Argonne National Laboratory, 9700 South Cass Ave., Argonne, IL 60439

27. F. Brouillard, Universite Catholique de Louvain, Dept. de Physique, FYAM, Chemin du Cyclotron 2, B-1348 Louvain-la-Neuve, Belgium

28. M. Capitelli, Centro di Studio Chimica dei Plasmi, Università de Bari, Piazza Umberto 1, I-Bari 70100, Italy

29. R.E.H. Clark, Atomic and Molecular Data Unit, P.O. Box 100, International Atomic Energy Agency, A-1400 Vienna, Austria

30. J. P. Colgan, Physics Department, Auburn University, Auburn AL 36849

31. D. P. Coster, Boltzmannstr 2, Munich, D-85748 Garching, Germany

32. M. D. Crisp, Office of Fusion Energy Sciences, Department of Energy, Germantown, MD 20874-1290

33. J. L. Delcroix, GAPHYOR, Laboratoire de Physique des Plasmas, Universite de Paris XI (ParisSud), 15, Rue G. Clemenceau, F-91405 Orsay Cedex, France

34. S. A. Eckstrand, Office of Fusion Energy Sciences, Department of Energy, Germantown, MD 20874-1290

35. T. E. Evans, 11439 Cloudcrest Dr,, San Diego, CA 92127-2027

36. I. Fabrikant, Department of Physics and Astronomy, University of Nebraska, 357 Behlen Lab, Lincoln NE 68588

37. U. Fantz, Lehrstuhl für Experimentelle Plasmaphysik, Memmingerstrasse 6, 86135 Augsburg, Germany

38. M. Finkenthal, Department of Physics and Astronomy, Johns Hopkins University, Bloomberg Center, Homewood Campus, Baltimore, MD 21218

39. W. Fritsch, Hahn-Meitner Institut Berlin GmbH, Bereich Theoretische Physik, Glienicker Strasse 100, D-14109 Berlin, Germany

40. T. Fujimoto, Department of Engineering Physics and Mechanics, Faculty of Engineering, Kyoto University, Oiwake-cho, Kitashirakawa, Yoshida-Honmachi, Sakyo-ku, Kyoto, 606-01, Japan

41. H. B. Gilbody, Department of Pure and Applied Physics, The Queen's University of Belfast, Belfast BT7 1NN, Northern Ireland, UK

42. D. C. Griffin, Department of Physics, Rollins College, 1000 Holt Ave., Winter Park FL 32789-4499

43. D. Humbert, GAPHYOR, Laboratoire de Physique des Plasmas, Universite de Paris XI (Paris-Sud), 
15, Rue G. Clemenceau, F-91405 Orsay Cedex, France

44. V. L. Jacobs, Code 6693, Naval Research Lab, 4555 Overlook Ave. SW, Washington DC 203755345

45. R. K. Janev, Macedonian Academy of Sciences and Arts, Skopje, Macedonia

46. C.F.F. Karney, Plasma Physics Laboratory, Princeton University, P.O. Box 451, Princeton, NJ 08543-0541

47. Takako Kato, National Institute for Fusion Science, Oroshi-cho, Toki, Gifu, 509-5292, Japan

48. Y.-K. Kim, Stop 8423, National Institute for Standards and Technology, 100 Bureau Dr., Gaithersburg, MD 20899-8423

49. S. Krasheninnikov, Department of Mechanical and Aerospace Engineering, University of California, San Diego, San Diego, CA 92093-0411

50. B. LaBombard, NW17-109, Massachusetts Institute of Technology, Plasma Science and Fusion Center, 175 Albany St., Cambridge, MA 02139

51. B. Lipschultz, MS NW17-103, Massachusetts Institute of Technology, 175 Albany St., Cambridge, MA 02139

52. A. Loarte, Max-Planck-Institut für Plasmaphysik, Boltzmannstrasse 2, D-85748 Garching, Germany

53. S. C. Luckhardt, University of California, San Diego, Bldg. EBU2, Room 460, 9500 Gilman Dr., Mail Code 417, La Jolla, CA 92093-0417

54. R. H. McKnight, Office of Fusion Energy Sciences, Department of Energy, Germantown, MD 20874-1290

55. T. J. Morgan, Department of Physics, Wesleyan University, Middletown, CT 06459

56. I. Murakami, Data and Planning Centre, National Institute for Fusion Science, 322-6, Oroshi-cho, Toki-shi, Gifu-ken, 509-52, Japan

57. R. E. Olson, Department of Physics, University of Missouri, Rolla MO 65401

58. R. A. Phaneuf, Department of Physics, University of Nevada, Reno, NV 89557-0058

59. A. Pigarov, Department of Mechanical and Aerospace Engineering, University of California, San Diego, 9500 Gilman Dr., San Diego, CA 92093-0411

60. M. S. Pindzola, Physics Department, Auburn University, Auburn AL 36849

61. S. Pitcher, NW17-111, Massachusetts Institute of Technology, Plasma Science and Fusion Center, 175 Albany St., Cambridge, MA 02139

62. G. D. Porter, 862 Adams Court, Livermore CA 94550

63. D. E. Post, L-038, Lawrence Livermore National Laboratory, P.O. Box 808, Livermore, CA 94551

64. D. Reiter, IPP, Forschungszentrum Jülich GmbH, D-52425 Jülich, Germany

65. M. E Rensink, L-637, Lawrence Livermore National Laboratory, University of California, Livermore, P.O. Box 808, Livermore CA 94551

66. T. D. Rognlien, L-630, Lawrence Livermore National Laboratory, University of California, Livermore, P.O. Box 808, Livermore CA 94551

67. E. A. Rohlfing, Office of Basic Energy Sciences, Department of Energy, Germantown, MD 208741290

68. W. L. Rowan, Fusion Research Center, University of Texas, Austin, TX 78712

69. D. N. Ruzic, University of Illinois at Urbana-Champaign, 103 S. Goodwin Ave., Urbana IL 61801

70. W. Sadowski, Office of Fusion Energy Sciences, Department of Energy, Germantown, MD 20874-1290

71. R. Schneider, Max-Planck-Institut für Plasmaphysik, Boltzmannstrasse 2, D-85748 Garching Germany

72. T. Shirai, Nuclear Data Centre, Physics Department, Japan Atomic Energy Research Institute (JAERI), 2-4 Shirakata Shirane, Tokai-mura, Naka-gun, Ibaraki-ken 319-11, Japan

73. P. C. Stancil, Department of Physics and Astronomy, University of Georgia, Athens, GA 306022451

74. P. C. Stangeby, Institute for Aerospace Studies, University of Toronto, 4925 Dufferin St., Downsview ON M3H 5T6 Canada

75. J. Stephens, Atomic and Molecular Data Unit, P.O. Box 100, International Atomic Energy Agency, A-1400 Vienna, Austria

76. D. P. Stotler, Princeton Plasma Physics Laboratory, Princeton University, P.O. Box 451, Princeton 
NJ 08543-0451

77. H. P. Summers, University of Strathclyde, Department of Physics and Applied Physics, 107

Rottenrow, Glasgow G4 0NG, United Kingdom

78. H. Takenaga, Japan Atomic Energy Research Institute, 801-1 Mukouyama Naka-machi, Naka-gun 311-0193 Japan

79. J. L. Terry, Bldg. NW17-176, Massachusetts Institute of Technology, 175 Albany St., Cambridge, MA 02139

80. E. W. Thomas, School of Physics, Georgia Institute of Technology, Atlanta, GA 30332-0430

81. M. R. Wade, General Atomics, P.O. Box 85608, San Diego, CA 92186-9784

82. J. M. Wadehra, Department of Physics and Astronomy, Wayne State University, Detroit MI 48202

83. J. Wang, Department of Physics and Astronomy, University of Georgia, Athens, GA 30602-2451

84. P. West, General Atomics, Fusion Group - Room: 13/470, P.O. Box 85608, San Diego, CA 921865608

85. W. Wiese, Atomic Physics Division 842.00, National Institute of Standards and Technology, Bldg 221, Room A-267, Gaithersburg, MD 20899-0001 